\title{
RAPID LOSS OF DUPLICATE GENE EXPRESSION BY NATURAL SELECTION
}

\author{
FRED W. ALLENDORF* \\ Genetics Research Unit, Queen's Medical Centre, University of Nottingham, Nottingham NG7 2UH
}

Received 24.v.79

\section{SUMmaRy}

Theoretical studies of the rate of fixation of null alleles at duplicate loci were carried out with both deterministic numeric analysis and computer simulations assuming an intermediate selective optimum of the number of active gene copies. A relatively rapid loss of duplicate gene expression occurs with this model under a wide variety of conditions, including incomplete recessiveness of the null allele and linkage. It differs from previous models of gene loss, which are based on mutation pressure, because the rate of gene loss is accelerated with increasing population size. Identification of the mode of selection responsible for the observed loss of gene duplication in polyploid fish is not possible at present. It appears, however, that the loss of duplicate gene expression occurs shortly following duplication. Once some structural or regulatory genetic divergence occurs, the duplicated loci become relatively stable.

\section{INTRODUCTXON}

THE use of isozymes as molecular probes of the genome has led to the detection of widespread gene duplication. Many of the enzymes that have been studied in plants and animals are coded by multiple loci. Harris (in press) has recently reviewed the extensive data available on human enzymes and concluded that 35 per cent of the enzymes studied are coded by multiple loci. In addition, several groups of both invertebrates and vertebrates are either polyploid or have undergone polyploidy in the evolutionarily recent past (White, 1978; Ohno, 1974). The importance of polyploidy has long been recognised in the plant kingdom; an estimated 47 per cent of all angiosperm species are polyploid (Grant, 1971). Gene duplications have also been shown to play an important role in the evolution of prokaryotes (Hartley, 1974). Gene duplication is thus a common evolutionary event.

Haldane (1933) first suggested that one of the two resultant duplicate loci may become non-functional through the accumulation, and eventual fixation, of deleterious (i.e. null) mutations at one locus of the duplicated pair while the other locus continued to perform the original function. Fisher (1935) showed that Haldane's model depends upon random genetic drift and that such gene loss does not occur in a deterministic model with infinite population size. Nei and Roychoudhury (1973) have further analysed this model showing that the fixation of a non-functional gene is highly dependent on population size, and that such loss of duplicate gene expression is likely only if the effective population size $(\mathcal{N})$ is smaller than 2000.

* Present address: Department of Zoology, University of Montana, Missoula, MT 59812 U.S.A. 
Fish of the families Salmonidae and Catastomidae provide evidence that loss of duplicate gene expression does often occur. There is good evidence that these families separately underwent tetraploidy approximately 50100 million years ago (Ohno, 1974). Tetraploid species have also evolved independently in the Cyprinidae and the Cobitidae (Ohno, 1974). Isozyme studies have shown that approximately 50 per cent of the expected duplicate genes following tetraploidy are apparently no longer expressed in these groups (Engel et al., 1975; Ferris and Whitt, 1977a; Ferris and Whitt, $1977 b$; Allendorf, 1978).

These results have stimulated further theoretical consideration of the loss of duplicate gene expression. Bailey et al. (1978) have used computer simulations to examine the expected rate of loss of duplicate gene expression when the double-recessive genotype is lethal and all the other genotypes have equal fitness. They conclude that the rate of loss is almost totally a function of population size and that the time until 50 per cent gene silencing is approximately $15 \mathcal{N}$. This conclusion is not in agreement with recent numeric and computer simulation results (Kimura and King, in press; Takahata and Maruyama, in press) that have shown that both population size and mutation rate have an important effect on the expected rate of loss of duplicate gene expression. Takahata and Maruyama also examined the expected rate of loss of duplicate gene expression with several other possible schemes of selection. All of these models share the essential feature that the loss of duplicate gene expression is caused by mutation pressure and genetic drift.

In this paper, I present a model in which the loss of duplicate gene expression is favoured by natural selection by assuming an intermediate optimum in the amount of active gene product. My objective is to present the evolutionary dynamics of this model, using both deterministic numeric analysis and a series of computer simulations to examine the effect of finite population size.

\section{THE MODEL}

\section{(i) Assumptions and definitions}

Consider in a random mating population two loci, each with two alleles, an active allele $(A, B)$ and a null allele $(a, b)$. The model assumes a recombination rate between loci of $r$ and unidirectional mutation from the active to the null allele with frequency $u$. The gametic frequencies in a given generation are defined as $x_{1}=$ freq $(A B), x_{2}=$ freq $(A B), x_{3}=$ freq $(a B)$, and $x_{4}=$ freq $(a b)$. The recursion equations connecting gametic frequencies between two generations $\left(x_{i}\right.$ and $\left.x_{i}^{\prime}\right)$ in the deterministic case have been presented by Karlin and McGregor (1971).

In a haploid population there are four phenotypes with the following fitnesses.

$$
\begin{array}{ccc} 
& B & b \\
A & 1-s & 1 \\
a & 1 & 0
\end{array}
$$

In a diploid population there are five phenotypic classes with the following fitnesses. 


$\begin{array}{cccc} & B B & B b & b b \\ A A & 1-s & 1-\frac{s}{2} & 1 \\ A a & 1-\frac{s}{2} & 1 & 1 \\ a a & 1 & 1 & 0\end{array}$

Thus, the homozygote for the null allele is lethal and there is some decrease in fitness when "extra" copies of the active allele are present.

\section{(ii) Simulations}

Changes in gametic frequencies were modelled in a diploid population by a strictly Monte Carlo simulation using different values of $s, u$, and $\mathcal{N}$ to examine the effects of genetic drift on the rate of gene loss. Two uniformly distributed (0-1) random numbers are used to select the two parental zygotes. A maximum of four more random numbers are used for each parental zygote to determine if recombination has occurred, to select a gamete, and to determine if mutation has occurred at either locus. A final random number is used to determine if the zygote formed by the union of the two gametes has survived. This process is repeated until $\mathcal{N}$ new zygotes are selected which then become the parental zygotes for the next generation.

\section{RESULtS}

\section{(i) Deterministic analysis}

The general behaviour of the model can be seen by examining the gene frequency trajectories shown in fig. 1. There are two stable equilibria and

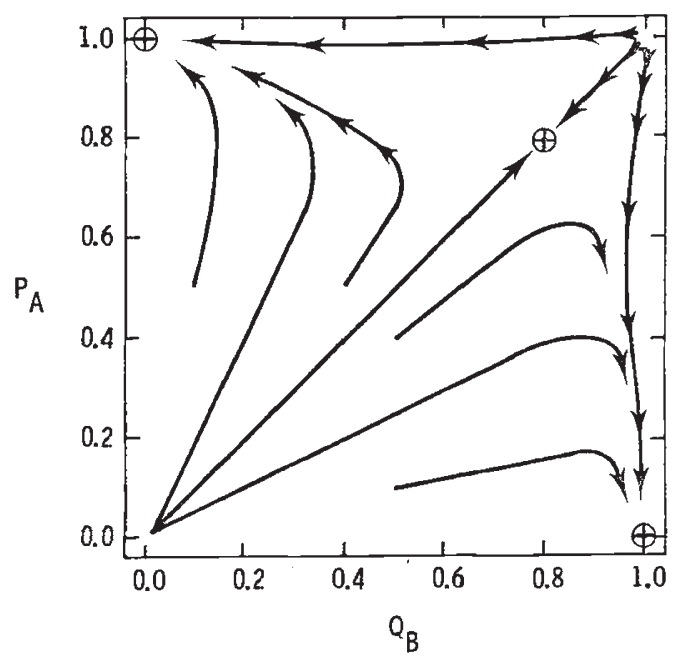

Fig. 1.-Gene frequency trajectories. Above the diagonal, $s=0 \cdot 10, u=10^{-5}$, and each arrow represents an interval of 25 generations. On and below the diagonal, $s=0 \cdot 02$, $u=10^{-5}$, and each arrow represents an interval of 100 generations. Equilibria are indicated by + . 
a line $\left(p_{A}=q_{B}\right)$ leading to an unstable equilibrium. The relationship $p_{A}=q_{B}$ will always lead to an equilibrium when $p_{A}=q_{B}$ for any scheme of selection and mutation for a duplicated locus system sharing the same two alleles at both loci. This follows directly from the recursion equations because whenever $x_{2}=x_{3}$, then $x_{2}^{\prime}=x_{3}^{\prime}$ will also be true. We know that

$$
p_{A}-q_{B}=\left(x_{1}+x_{2}\right)-\left(x_{1}+x_{3}\right)=x_{2}-x_{3}
$$

Therefore, when $x_{2}=x_{3}, p_{A}-q_{B}=0$ and $p_{A}=q_{B}$.

The equilibrium on the line defined by $p_{A}=q_{B}$ is unstable so that in finite populations there is global convergence towards fixation of one of the null alleles $(a$ or $b)$, depending upon the initial state. Thus, the population will evolve toward a stable equilibrium at one locus so that in a diploid population either

or

$$
p_{a}=1 \text { and } q_{b}=\sqrt{u}
$$

$$
p_{a}=\sqrt{u} \text { and } q_{b}=1
$$

The rate of loss of duplicate gene expression (defined as either $p_{A}$ or $q_{B}$ being less than 0.01 ) depends upon $u, s$, and the initial state. We are interested in the conditions after gene duplication by polyploidy. Newly derived tetraploid populations presumably are the descendants of one or very few individuals. We thus assume the initial frequencies of the null allele at both loci are zero, except in the deterministic analysis where the initial frequency of either $p_{a}$ or $q_{b}$ was decreased by 0.01 to move off the line leading to the unstable equilibrium.

\section{TABLE 1}

Number of generations until loss of duplicate gene expression in the deterministic case. Loss is defined to occur when the frequency

\begin{tabular}{|c|c|c|c|c|c|}
\hline & & & & & \\
\hline & $s$ & $10^{-8}$ & $10^{-4}$ & $10^{-5}$ & $10^{-6}$ \\
\hline Haploid case & $0 \cdot 10$ & 90 & 88 & 88 & 88 \\
\hline & 0.02 & 464 & 459 & 458 & 458 \\
\hline Diploid case & $0 \cdot 10$ & 213 & 189 & 180 & 177 \\
\hline & 0.02 & 1062 & 974 & 936 & 922 \\
\hline
\end{tabular}
of the null allele exceeds 0.99 at one of the two loci. The initial frequencies of the null allele are $\mathrm{p}_{\mathrm{a}}=0.00$ and $\mathrm{p}_{\mathrm{b}}=0.01$

Table 1 shows that the fixation of one of the null alleles occurs quite rapidly (approximately 1000 generations) in the deterministic case, even with very small selection coefficients. The fixation of the null allele occurs more rapidly for larger values of $s$. Perhaps surprisingly, the fixation of the null allele occurs more slowly as its mutation rate is increased. This effect results from fixation occurring more rapidly the closer the frequencies stay to the one-locus boundary. An increased mutation rate at both loci tends to move the conditions away from the edges and thus delays loss. This effect is not important, however, in finite populations where the major retarding factor is the initial establishment of the null allele. 


\section{(ii) Simulations}

A series of simulations for a diploid population were performed using four different population sizes $(50,200,1000,2000)$ in association with the two fitness sets and the four mutation rates used in the deterministic model. Twenty-five separate simulations were completed using all possible combinations of the three parameters. These results agree well with the deterministic model when using large population sizes and high mutation rates (see table 2).

TABLE 2

Simulation results of the time required for the loss of duplicate gene expression in the diploid case. Loss is defined to occur as in table 1. Each number is the middle value among 25 repeats, and thus represents the number of generations until 50 per cent loss of duplicate gene expression $\left(\mathrm{T}_{0.5}\right)$. The initial null allele frequencies are $\mathrm{p}_{\mathrm{a}}=\mathrm{p}_{\mathrm{b}}=0$

\begin{tabular}{|c|c|c|c|c|c|}
\hline & & \multicolumn{4}{|c|}{$t$} \\
\hline & $\mathcal{N}$ & $10^{-3}$ & $10^{-4}$ & $10^{-5}$ & $10^{-6}$ \\
\hline \multirow[t]{4}{*}{$s=0.10$} & 50 & 155 & 345 & 2304 & 16933 \\
\hline & 200 & 168 & 171 & 610 & 4854 \\
\hline & 1000 & 210 & 209 & 226 & 3338 \\
\hline & 2000 & 153 & 176 & 203 & 490 \\
\hline \multirow[t]{4}{*}{$s=0.02$} & 50 & 309 & 1334 & 11868 & 45224 \\
\hline & 200 & 553 & 784 & 2694 & 13714 \\
\hline & 1000 & 534 & 777 & 1275 & 5112 \\
\hline & 2000 & 495 & 644 & 938 & 2289 \\
\hline
\end{tabular}

The number of generations until 50 per cent loss $\left(T_{0.5}\right)$ appears to be approximately equivalent for a given value of $\mathcal{N} u$. For example, compare equal values of $\mathcal{N} u$ for $\mathcal{N}=200$ and 2000. A further series of simulations confirmed that the rate of loss is approximately the same for a given value of $\mathcal{N} u$ with different size populations (unpublished results). A more extensive series of simulations are used in fig. 2 and 3 to describe the rate of gene loss for different values of $\mathcal{N u}$.

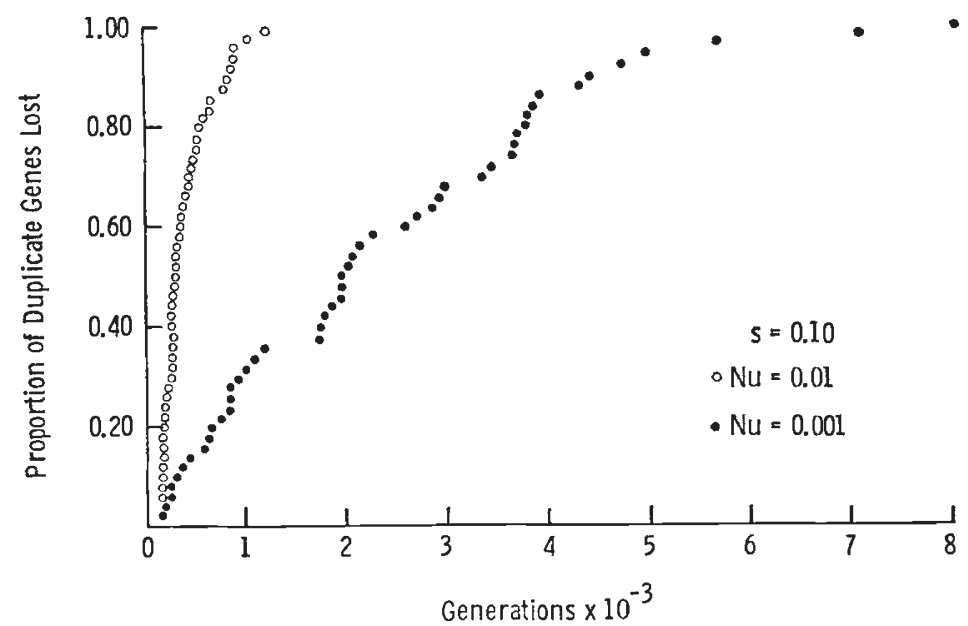

FIG. 2.-Simulation results showing the rate of loss of duplicate gene expression with $s=0 \cdot 10$ for different values of $\mathcal{N} u ;(\mathcal{N}=1000)$. 


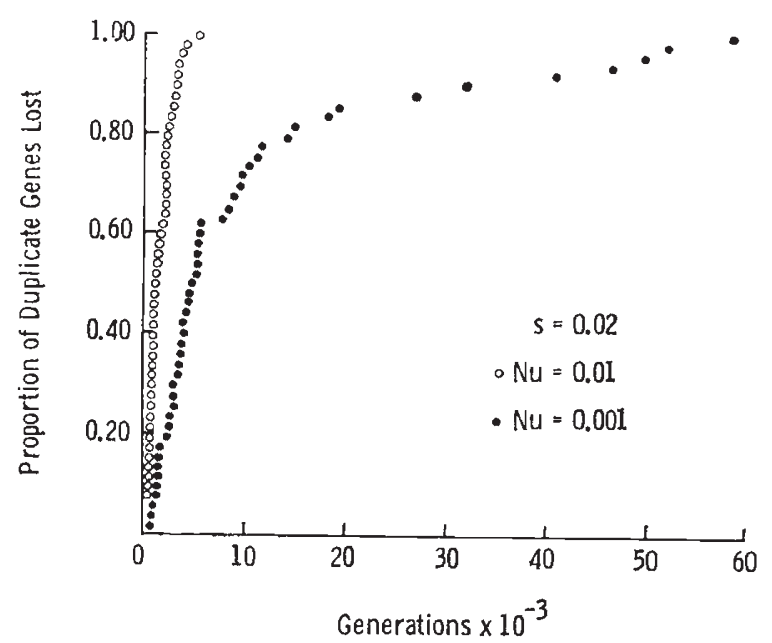

FIG. 3.-Simulation results showing the rate of loss of duplicate gene expression with $s=0.02$ for different values of $\mathcal{N} u ;(\mathcal{N}=1000)$.

\section{(iii) Linkage}

The effect of linkage can be seen by analysing the case of absolute linkage in the haploid case. This situation is equivalent to a single locus having four alleles that are the four gamete types. Once the frequency of the less fit nonlethal "allele" $(A B)$ reaches zero, a neutral equilibrium is obtained where $x_{2}+x_{3}=1 \cdot 0$. It is no longer reasonable to define gene loss as either $p_{a}$ or $q_{b}$ being less than $0 \cdot 1$. When $x_{1}=x_{4}=0, p_{a}=x_{3}$ and $p_{b}=x_{4}$, and for all values of $p_{a}+p_{b}=1.0$ there is effective gene loss because each individual carries only one copy of the active allele.

This same effect occurs in the diploid case. In the case of absolute linkage, there are four alleles with the following relationships of average fitness

$$
w_{A B}<w_{A b}=w_{a B}>w_{a b}
$$

An equilibrium will be reached when $x_{1}=0$, so that the gamete $a b$ is at selection-mutation balance

$$
x_{4}=\sqrt{u}
$$

and the gametes $A b$ and $a B$ are in a neutral equilibrium

$$
x_{2}+x_{3}=1-\sqrt{u}
$$

Linkage apparently does not strongly alter the rate of loss of duplicate gene expression, if we define loss to occur when the frequency of the phenotype having more than the optimal copies of the active allele to be less than $0 \cdot 01$. This is confirmed both by deterministic results (table 3 ) and by simulations in the diploid case (table 4). The expected loss of expression occurs slightly quicker with absolute linkage as compared to random assortment. There are intermediate values of recombination, however, at which duplicate gene expression persists somewhat longer than with either extreme of recombination. Departures from linkage equilibrium are found following loss of expression (see table 4 ) only with very tight linkage $(r \leqq 0 \cdot 01)$. 
TABLE 3

\begin{tabular}{|c|c|c|c|}
\hline \multicolumn{4}{|c|}{$\begin{array}{l}\text { Number of generations until loss of duplicate gene } \\
\text { expression for different rates of recombination in } \\
\text { the deterministic case. Loss is defined to occur } \\
\text { when the frequency of genotypes with more than } \\
\text { the optimal number of active gene copies is less } \\
\text { than } 0.01 \text {. The initial frequencies of the null } \\
\text { allele are } \mathrm{p}_{\mathrm{a}}=0.00 \text { and } \mathrm{p}_{\mathrm{b}}=0.01 ; \mathrm{u}=10^{-5}\end{array}$} \\
\hline$r$ & $s$ & Haploid & Diploid \\
\hline 0.500 & $\begin{array}{l}0 \cdot 10 \\
0 \cdot 02\end{array}$ & $\begin{array}{r}88 \\
456\end{array}$ & $\begin{array}{r}192 \\
1000\end{array}$ \\
\hline $0 \cdot 100$ & $\begin{array}{l}0 \cdot 10 \\
0 \cdot 02\end{array}$ & $\begin{array}{r}88 \\
456\end{array}$ & $\begin{array}{r}193 \\
1002\end{array}$ \\
\hline $0 \cdot 050$ & $\begin{array}{l}0 \cdot 10 \\
0 \cdot 02\end{array}$ & $\begin{array}{r}88 \\
457\end{array}$ & $\begin{array}{r}195 \\
1005\end{array}$ \\
\hline 0.010 & $\begin{array}{l}0 \cdot 10 \\
0 \cdot 02\end{array}$ & $\begin{array}{r}88 \\
476\end{array}$ & $\begin{array}{r}197 \\
1059\end{array}$ \\
\hline 0.005 & $\begin{array}{l}0 \cdot 10 \\
0 \cdot 02\end{array}$ & $\begin{array}{r}88 \\
480\end{array}$ & $\begin{array}{r}194 \\
1151\end{array}$ \\
\hline 0.001 & $\begin{array}{l}0 \cdot 10 \\
0 \cdot 02\end{array}$ & $\begin{array}{r}88 \\
461\end{array}$ & $\begin{array}{r}190 \\
1220\end{array}$ \\
\hline 0.000 & $\begin{array}{l}0.10 \\
0.02\end{array}$ & $\begin{array}{r}88 \\
451\end{array}$ & $\begin{array}{l}189 \\
964\end{array}$ \\
\hline
\end{tabular}

TABle 4

Simulation results of the time to loss of duplicate gene expression for different values of recombination in the diploid case. Loss is defined to occur as in table 3. Each number is the middle value among 25 repeats $\left(\mathrm{T}_{0.5}\right) . \quad \mathrm{P}$ is the proportion of the cases in which neither $\mathrm{p}_{\mathrm{a}}$ nor $\mathrm{p}_{\mathrm{b}}$ was greater than 0.99 at the point of loss of duplicate gene expression. The initial null allele frequencies are $\mathrm{p}_{\mathrm{a}}=\mathrm{p}_{\mathrm{b}}=0$, and $\mathrm{Nu}=0.1(\mathrm{~N}=1000) ; \mathrm{S}=0.02$

$\begin{array}{ccc}r & T_{0.5} & P \\ 0.500 & 777 & 0 \\ 0.100 & 755 & 0 \\ 0.050 & 875 & 0 \\ 0.010 & 923 & 0.16 \\ 0.005 & 781 & 0.28 \\ 0.001 & 677 & 0.48 \\ 0.000 & 772 & 0.56\end{array}$

\section{(iv) Incomplete recessive case}

Takahata and Maruyama (in press) show that even small deleterious effects of the null allele in genotypes with only one active allele can greatly retard the rate of loss of duplicate gene expression expected with the mutation pressure model of gene loss. The presence of incomplete recessiveness is not important with the selective model of gene loss. The critical period for determining the rate of gene loss is the establishment of the null allele at one of the loci. Once this occurs, selection will act to fix the null allele, even if genotypes with only one active allele have greatly reduced fitness. Such a reduction in fitness will not affect the initial establishment of the null allele 
because genotypes with only a single active allele will be extremely rare in the population until the null allele is established.

Once sufficient time has elapsed for establishment of the null allele at one or both loci, the effect of reduced fitness of the $A a b b$ and $a a B b$ genotypes is only to change the path of gene frequencies and not to change the actual rate of loss. The lower the fitness of $A a b b$ or $a a B b$ the closer the gene frequencies remain to the one-locus boundary. Thus, even if the genotypes with one active allele are lethal, the rate of loss of duplicate gene expression is not significantly changed.

\section{Discussion}

\section{(i) Biological basis of the model}

This model of gene loss depends upon genotypes with both the active and null allele having the highest fitness. There are several different possible causes of this effect. There is some evidence that many recessive lethals have a slightly beneficial effect in heterozygotes (e.g., Wallace, 1966), although it does appear that overdominant lethal mutations are actually rather rare (Simmons and Crow, 1977). Any recessive lethal mutation that is overdominant with respect to fitness will fit the present model of gene loss.

The quantity of the active enzyme produced may also be important for fitness. Any case in which there was an intermediate optimum in activity may fit the present model. Latter (1975) and also Fincham (1972) have argued that "natural selection for a fixed optimal level of activity in the prevailing environment" could in fact be responsible for much of the enzyme polymorphism detected at individual loci. Any effect of such an optimum level of activity should be stronger at a duplicated locus which has a much larger range of potential activities of genotypes.

There are at least two additional effects that could bring about selection for an optimal amount of activity at a duplicated locus. One is simple metabolic efficiency. Some individual enzymes represent a significant proportion of the total soluble protein concentration of certain tissues. The overproduction of certain enzymes may therefore cause selection for reduced production of gene product. Some polyploids have been found to have greatly increased amounts of enzyme activity relative to their diploid counterparts (Levin et al., 1979 and references therein).

The second mechanism that could cause selection for an intermediate optimum of enzyme activity is the interaction with other enzymes in the same metabolic pathway. Too much enzyme activity could produce an excess of a reaction product that could adversely affect the function of a particular pathway. The loss of duplicate gene expression for one enzyme in a pathway may thus cause a "domino" effect on the surrounding enzymes. It would be of interest to look at the set of enzymes that have lost duplicate gene expression in particular catastomid and salmonid species to detect any such effect.

There is yet another mechanism that could cause the fitness scheme assumed in this model. The loci resulting from a duplication often acquire different functions. For example, many of the oxidoreductases share homologies that suggest they are descendants of a single ancestral gene that have evolved different substrate specificities. A mutation may occur at one of the 
duplicate loci so that this locus produces an enzyme that improves fitness, yet no longer performs the original function. Thus, the expression of this locus would be effectively lost as determined by the empirical definition of performing the original function. This mechanism is analogous to Wright's (1964) model of the loss of an unnecessary morphological feature by pleiotropic effects, as compared to the simple accumulation of non-functional alleles by mutation pressure and genetic drift.

There is empirical support for the present model of loss of duplicate gene expression in both prokaryotes and eukaryotes. Andrews and Hageman (1976) found that five strains of Escherichia coli producing excess lac operon protein had a $2 \cdot 0-2 \cdot 5$ per cent reduction in growth rate. In addition, Hartley et al. (1976) successfully selected for strains of Klebsiella aerogenes carrying a duplication of the ribitol dehydrogenase locus. Once the selection pressure for excessive production of this enzyme was removed, however, they found evidence of selection against the strains with the duplication.

Roberts and Baker (1973) and Baker (1975) have studied null alleles at a tandem duplication for an $\alpha$-napthyl acetate-specific esterase locus in Drosophila montana. They found four tightly linked genes that result from two tandem duplication events. The two loci originating from the primary duplication event apparently diverged in function prior to the second duplication so that there are two functionally distinct genes that have in turn been duplicated. In several separate populations, there is strong linkage disequilibria between null alleles within both pairs of functional genes, resulting in a large deficit of chromosomes with more than two copies of an active allele. They conclude that too much enzyme is detrimental to fitness and there has been selection for only one active allele at each functional $\alpha$-esterase gene per chromosome.

\section{(ii) Expected rate of loss of duplicate gene expression}

This model predicts a relatively rapid loss of duplicate gene expression under a wide range of conditions. The expected rate of loss increases as population size increases, in striking contrast to previously described models of gene loss. Takahata and Maruyama (in press) conclude that the expected value of $T_{0.5}$ with a variety of selection schemes in the strictly mutation pressure case is approximately $10 \mathcal{N}$. Thus if $\mathcal{N}=2000$ and $u=10^{-5}$, the mutation pressure models predict a $T_{0.5}$ of approximately 20,000 generations, as compared to 900 generations predicted by the present model with $s=0.02$. As $\mathcal{N}$ increases these predictions will further diverge.

The present mathematical treatment ignores the genetic mechanisms responsible for loss of duplicate gene expression. A variety of potential mechanisms can be imagined: suppression of transcription or translation, production of an inactive gene product, a chain-terminating mutation, or a deletion. Intensive screening of null mutations for alcohol dehydrogenase and xanthine dehydrogenase in $D$. melanogaster has detected many such mutants, almost all of which have been found to be structural mutations Gelbart et al., 1976; Schwartz and Sofer, 1976). An understanding of the mechanisms responsible for the observed loss of duplicate gene expression would be valuable but will not necessarily allow discrimination between the alternative models of loss. All of these mechanisms for loss are functionally 
equivalent in the mutation pressure model. Some of these mechanisms are less likely, however, to fit the selective loss model. Nevertheless, even the "wasteful" production of inactive gene product can fit this model in the case of selection for an optimum amount of activity or in the case of acquiring a completely new function.

The rapid loss of duplicate gene expression predicted by the selective model does not mean that the evolution of new gene function by duplicates is necessarily less likely. The duplicated genes themselves can be retained and may regain activity by subsequent mutations. Indeed, Koch (1972) has argued that such a period of "suppression" of gene expression actually allows more rapid evolution of new enzymatic activity.

We cannot decide at present which model of the loss of duplicate gene expression is more likely to have brought about the observed losses in polyploid fish. The 35-100 million year period since the induction of polyploidy is long enough for the loss of duplicate gene expression to have occurred at almost all loci, either by the present selection scheme or by mutation pressure in the completely recessive case. The prolonged retention of duplicate gene expression may be explained by null alleles having a deleterious effect in all genotypes (Takahata and Maruyama, in press). This explanation assumes that loss of duplicate gene expression has been occurring at a uniform rate until the present. However, all four groups of fish which have undergone tetraploidy at different times show loss of approximately 50 per cent of their duplicate loci. The most extreme group is the cobitid loaches that show loss of an estimated 75 per cent of their duplicate genes (Ferris and Whitt, 1977b). This group is the most recent tetraploid, originating approximately 35-40 million years ago, as compared to the estimated 100 million years ago in the salmonids (Bailey et al., 1978). Thus, there is no evidence of increased gene loss with increasing time since tetraploidy among these groups of fish.

An important factor that must be considered is the regulatory divergence of duplicated loci. Those duplicate loci that are retained usually show different tissue expression. Once such differentiation occurs the fitness models considered here are no longer appropriate. For example, if only one of the duplicated loci is expressed in liver tissue, then a homozygous null at that locus is unlikely to be compensated for by active alleles at the other locus. Such a mutation that restricts the expression of one locus to certain tissues may be selected for in the fitness scheme presented here. Other subsequent similar mutations may also be favoured, as long as at least one locus is expressed in every tissue. Therefore, both the retention of duplicate genes with different tissue expression and the loss of duplicate gene expression can result from the same selective pressures.

Another possible explanation for the prolonged retention of some duplicate loci in salmonids and catastomids is the possibility that two active alleles may confer a selective advantage when both are present in a single individual. Natural selection would then act to produce fixed "heterozygotes" for these two active alleles, just as in the selection scheme considered in this article. Such a system would tend to maintain duplicate gene expression indefinitely at some loci.

Thus, we do not expect to see the eventual loss of all of the "extra" genes created by gene duplication. Once either structural or regulatory divergence has occurred at these loci, they should become stable and not be susceptible to the models of loss of expression we have considered. This 
conclusion finds support in the remarkable stability of the multiple loci originating from ancient gene duplication events coding for a variety of enzymes in vertebrates, e.g. lactate dehydrogenase (Markert et al., 1975), creatine kinase (Fisher and Whitt, 1978), and phosphoglucose isomerase (Avise and Kitto, 1973).

The "fate" of a duplicated locus is apparently determined shortly following duplication. The evidence from polyploid fish indicates that approximately 50 per cent of the duplicated loci are lost relatively rapidly after duplication. If however, structural or regulatory divergence occurs between the loci, they are then genetically distinct, and therefore much less susceptible to the loss of duplicate gene expression.

Acknowledgments. - I thank Professor Bryan Clarke for his suggestions and for providing facilities during my stay in the Genetics Research Unit, Bill Dunlap for commenting on the manuscript, and Drs M. Kimura and N. Takahata for kindly providing me with preliminary copies of their papers. I also thank Dr David Parkin of the Genetics Department and the staff of the Cripps Computer Center for generously providing the facilities and time to complete the simulations presented here. This work was done while I was supported by a NATO Fellowship from the National Science Foundation.

\section{REFERENCES}

ALLENdoRF, F. W. 1978. Protein polymorphism and the rate of loss of duplicate gene expression. Nature, 272, 76-78.

ANDREWS, K. J., AND HEgeman, G. D. 1976. Selective disadvantage of non-functional protein synthesis in Escherichia coli. F. Mol. Evol., 8, 317-328.

Avise, J. C., AND kITTO, G. B. 1973. Phosphoglucose isomerase gene duplication in the bony fishes: an evolutionary history. Biochem. Genet.; 8, 113-132.

BAILEY, G. S., PUlter, R. T. M., AND stOCKWELl, P. A. 1978. Gene duplication in tetraploid fish: model for gene silencing at unlinked duplicated loci. Proc. Nat. Acad. Sci. U.S.A., $75,5576-6579$.

BAKER, W. K. 1975. Linkage disequilibrium over space and time in natural populations of Drosophila montana. Proc. Nat. Acad. Sci. U.S.A., 72, 4095-4099.

ENGEL, W., SCHMIDTKE, J., AND WOLF, U. 1975. Diploid-tetraploid relationships in teleostean fishes. In Isozymes, Vol. IV: Genetics and Evolution, ed. C. L. Markert. Academic Press, New York.

FERRIS, s. D., AND WHITT, G. s. 1977a. Loss of duplicate gene expression after polyploidisation. Nature, 265, 258-260.

FERRIS, s. D., AND WHITT, G. s. 1977b. Duplicate gene expression in diploid and tetraploid loaches (Cypriniformes, Cobitidae). Biochem. Genet., 15, 1097-1112.

FINCHAM, J. R. S. 1972. Heterozygous advantage as a likely general basis for enzyme polymorphisms. Heredity, 28, 387-391.

Fisher, R. A. 1935. The sheltering of lethals. Amer. Natur., 69, 446-455.

FISHER, S. E., AND WHITT, G. s. 1978. Evolution of isozyme loci and their differential tissue expression. F. Mol. Evol., 12, 25-55.

gelbart, w., MCCARroN, M., AND GHOvNick, A. 1976. Extension of the limits of the XDH structural element in Drosophila melanogaster. Genetics, 84, 211-232.

Grant, v. 1971. Plant Speciation. Columbia University Press.

HALDANE, J. B. S., 1933. The part played by recurrent mutation in evolution. Amer. Natur., 67, 5-19.

HARRIs, H. (in press). Multi-locus enzymes in man. CIBA Foundation Symposium, No. 66 (new series).

HARTLEy, B. s. 1974. Enzyme families. In Evolution in the Microbial Worlds, eds. M. J. Carlile and J. J. Skehel. Cambridge University Press.

HARTLEy, B. S., AltosaAR, I., DOTHIE, J. M., AND NEUberger, M. s. 1976. Experimental evolution of a xylitol dehydrogenase. Proc. Third Fohn Innes Symp., 191-200.

KARLIN, s., AND MCGREgor, J. 1971. On mutation selection balance for two-locus haploid and diploid populations. Theoret. Pop. Biol., 2, 60-70. 
KIMURA, M., AND KING, J. L. (in press). Fixation of a deleterious allele at one of two "duplicate" loci by mutation pressure and random drift. Proc. Nat. Acad. Sci. U.S.A.

косн, А. L. 1972. Enzyme evolution. I. The importance of untranslatable intermediates. Genetics, 72, 297-316.

LATTER, B. D. H. 1975. Enzyme polymorphisms: gene frequency distributions with mutation and selection for optimal activity. Genetics, 79, 325-331.

LEVIN, D. A., TORRES, A. M., AND LEVY, M. 1979. Alcohol dehydrogenase activity in diploid and autotetraploid Phlox. Biochem. Genet., 17, 35-42.

MARKERT, c. L., Shaklee, J. B., AND Whitt, G. S. 1975. Evolution of a gene. Science, 189, 102-114.

NEI, M., AND ROYChOUdhury, A. K. 1973. Probability of fixation of non-functional genes at duplicate loci. Amer. Natur., 107, 362-372.

ohno, s. 1 974. Protochordata, Cyclostomata, and Pisces. Gebrüder-Borntraeger, Stuttgart.

ROBERTS, R. M., AND BAKER, w. K. 1973. Frequency distribution and linkage disequilibrium of active and null esterase isozymes in natural populations of Drosophila montana. Amer. Natur., 107, 709-726.

SCHWARTz, M., AND SOFER, w. 1976. Alcohol dehydrogenase-negative mutants in Drosophila: defects at the structural locus. Genetics, 83, 125-136.

simmons, M. J., AND CROW, J. F. 1977. Mutations affecting fitness in Drosophila populations. Ann. Rev. Genet., 11, 49.78.

takahata, N., ANd maruyama, T. (in press). Polymorphism and loss of duplicate gene expression: theoretical study and its applications. Proc. Nat. Acad. Sci. U.S.A.

WALLACE, B. 1966. Distance and the allelism of lethals in a tropical population of Drosophila melanogaster. Amer. Natur., 100, 565-578.

WHITE, M. J. D. 1978. Modes of Speciation. W. H. Freeman, San Francisco.

WRIGHT, s. 1964. Pleiotropy in the evolution of structural reduction and of dominance. Amer. Natur., 98, 65-69. 